\title{
Overexpression of zinc finger protein 384 (ZNF 384), a poor prognostic predictor, promotes cell growth by upregulating the expression of Cyclin D1 in Hepatocellular carcinoma
}

\author{
Lifeng He${ }^{1}$, Xiaoxiao Fan ${ }^{1}$, Yirun $\mathrm{Li}^{1}$, Mingming Chen ${ }^{1}$, Bin Cui ${ }^{3}$, Guoqiao Chen ${ }^{1}$, Yili Dai ${ }^{1}$, Daizhan Zhou ${ }^{4}$, \\ Xiaotong $\mathrm{Hu}^{2}$ and Hui Lin ${ }^{1,2}$
}

\begin{abstract}
Hepatocellular carcinoma (HCC) is a highly heterogeneous, multigene-driven malignant tumor. ZNF384 is an overexpressed gene with a high frequency of alteration in HCC, but research on the function of ZNF384 in HCC is lacking. In this study, the expression level of ZNF384 in HCC was analyzed through immunohistochemical (IHC) staining, Western blot analysis and GRT-PCR. We also generated ZNF384 knockdown and knockout HCC cell lines using short hairpin RNA (shRNA) and CRISPR/Cas9 systems. MTS, colony formation, and 5-ethynyl-20-deoxyuridine (EdU) assays; flow cytometry; and a xenograft mouse model were used to evaluate the effects of ZNF384 on cell proliferation. Western blot analysis, a dual luciferase reporter assay and a ChIP assay were performed to explore the potential mechanism. We found that overexpression of ZNF384 in HCC and elevated expression of ZNF384 in HCC tissues was significantly correlated with tumor recurrence $(P=0.0097)$. Kaplan-Meier survival analysis revealed that high expression levels of ZNF384 were correlated with poor overall survival $(P=0.0386)$. Downregulation of ZNF384 expression suppressed HCC cell proliferation by inhibiting the expression of Cyclin D1. These findings suggest that ZNF384 tends to act as an oncogene in the development of HCC. ZNF384 promotes the proliferation of HCC cells by directly upregulating the expression of Cyclin D1 and might serve as a prognostic predictive factor for HCC patients.
\end{abstract}

\section{Introduction}

Hepatocellular carcinoma (HCC) is a heterogeneous disease with a poor 5 -year survival rate of less than $10 \%{ }^{1}$. Multiple risk factors involve in the initiation and progression of HCC including HBV and/or HCV infections,

\footnotetext{
Correspondence: Xiaotong Hu (hxt_hz@zju.edu.cn) or

Hui Lin (369369@zju.edu.cn)

'Department of General Surgery, Sir Run Run Shaw Hospital, School of Medicine, Zhejiang University, Hangzhou, China

${ }^{2}$ Biomedical Research Center, Sir Run Run Shaw Hospital, School of Medicine, Zhejiang University, Hangzhou, China

Full list of author information is available at the end of the article.

These authors contributed equally: Lifeng $\mathrm{He}$, Xiaoxiao Fan

Edited by S. Tait
}

chronic alcohol consumption, metabolic syndrome, obesity and diabetes which contribute to the complex and extraordinarily heterogeneous characteristics of $\mathrm{HCC}^{2}$. In the era of advocating individualized treatment of cancers, the tumor heterogeneity greatly impedes the development of HCC molecular targeted drugs ${ }^{3,4}$. Genomic studies about HCC have depicted a map of molecular alterations in HCC. Regrettably, the targetable driven genes for treatment and the biomarkers for response of the targeted treatment is still mysterious ${ }^{5,6}$. As a result, the efficacy of targeted therapy including the sorafenib and regorafenib for the treatment of HCC is not satisfactory, especially in Chinese population ${ }^{7,8}$. It pushed us to explore the role of

\section{(c) The Author(s) 2019}

(c) (i) Open Access This article is licensed under a Creative Commons Attribution 4.0 International License, which permits use, sharing, adaptation, distribution and reproduction cc in any medium or format, as long as you give appropriate credit to the original author(s) and the source, provide a link to the Creative Commons license, and indicate if changes were made. The images or other third party material in this article are included in the article's Creative Commons license, unless indicated otherwise in a credit line to the material. If material is not included in the article's Creative Commons license and your intended use is not permitted by statutory regulation or exceeds the permitted use, you will need to obtain permission directly from the copyright holder. To view a copy of this license, visit http://creativecommons.org/licenses/by/4.0/. 
genes that have changed in $\mathrm{HCC}$ sequencing research in the development of HCC.

ZNF384 gene encodes a C2H2-type zinc finger protein which functions as a transcription factor regulates the transcription of the extracellular matrix genes ${ }^{9}$. The previous studies reported that ZNF384 fused with the TET family genes, including Ewing sarcoma breakpoint region 1 (EWSR1) gene, TATA box binding protein-associated factor (TAF15) and transcription factor 3 (TCF3) and played an important role in $\mathrm{ALL}^{10,11}$. Sakuma et al. ${ }^{12}$ found that the over expression of ZNF384 would promote the migration of melanoma cells. Mori et al. ${ }^{13}$ demonstrated that ZNF384 bound to APOBEC3B (A3B) promoter and functions as a modulator of $\mathrm{A} 3 \mathrm{~B}$ expression in cervical cancer. In addition, The Cancer Genome Atlas (TCGA) and Oncomine databases suggest that ZNF384 is an overexpressed gene with high frequency alterations in HCC. Although various evidences indicated that ZNF384 might be a potential oncogene, which promotes the occurrence and development of cancers, the research about ZNF384 in HCC is vacant.

In this study, we first identified the role of ZNF384 in the initiation and progression of HCC. We confirmed the overexpression of ZNF384 in HCC tissues. In addition, we characterized the oncogenes role of ZNF384 in HCC and explored the potential mechanism of ZNF384 in regulating cell proliferation in vivo and in vitro. Our results showed that ZNF384 promote tumor growth and may be a novel prognostic marker in HCC.

\section{Results}

Overexpression of ZNF384 in HCC tissues and cell lines

To explore the potential role played by ZNF384 in the development of HCC, we obtained genetic information for ZNF384 in HCC from the TCGA database. We found that the rate of genetic alteration of ZNF384 was as high as $9.4 \%$ and that mRNA upregulation accounted for $70.6 \%$ of these alterations (Fig. 1a). We also found that ZNF384 expression was significantly increased in HCC tissues compared to that in adjacent normal liver tissues in the TCGA RNA-seq database (Fig. 1b). The protein expression of ZNF384 was further assessed in HCC samples and corresponding adjacent normal tissues (ANT) by IHC staining. These results showed that most HCC samples exhibited higher protein expression of ZNF384 than ANT (Fig. 1c, d). Figure 1e, f also show that most HCC samples had elevated ZNF384 protein expression.

\section{High expression of ZNF384 in HCC indicates a worse prognosis}

The association of ZNF384 expression with overall survival was analyzed via Kaplan-Meier analysis in 71 patients with complete follow-up information. The high expression of ZNF384 predicted poorer prognosis in HCC patients $(P=0.0386$, Fig. $1 \mathrm{~g})$. The high expression of ZNF384 was closely related to the recurrence of HCC $(P=0.0097)$. We also observed that high expression of ZNF384 always correlated with shorter tumor-free survival time in patients $(P=0.0016$, Fig. 1g). The detailed patient information is shown in Table 1.

\section{Downregulated expression of ZNF384 inhibits HCC cells proliferation through $\mathrm{G} 1 / \mathrm{S}$ phase transition arrest}

To explore the role of ZNF384 in the development of HCC, we first verified the expression level of ZNF384 in liver cancer cell lines in order to select appropriate HCC cell lines. The results showed that ZNF384 was expressed at high levels in all HCC cell lines. Therefore, we selected HCCLM3, PLC/PRF/5, and Huh7 cells for the next experiment. We generated endogenous ZNF384 knockdown cell lines via shRNA in HCCLM3 and PLC/PRF/5 cells (Fig. 2a). MTS assays revealed that silencing ZNF384 in HCCLM3 and PLC/PRF/5 cells markedly inhibited cell growth (Fig. 2b). Similarly, ZNF384 knockdown reduced the colony formation ability of the HCCLM3 and PLC/ PRF/5 cell lines (Fig. 2c, d). The results of the MTS and colony formation assays indicated that ZNF384 promoted cell growth. The effect of the ZNF384 protein on the cell cycle was analyzed via EdU immunofluorescence and flow cytometry, and the EdU immunofluorescence results showed that the proportion of cells in $S$ phase was decreased when the expression of ZNF384 was silenced (Fig. 2e). In addition, the flow cytometric analysis results suggested that the cell cycle was arrested at the G1/S phase transition when ZNF384 expression was silenced (Fig. 2f).

To verify that phenotypic changes in cell proliferation were indeed caused by the reduced expression of ZNF384 rather than by off-target effects, we designed single guide RNAs (sgRNAs) targeting the second exon of the ZNF384 gene (Fig. 3a) and generated a ZNF384 knockout Huh7 cell line via the CRISPR/Cas9 technique (Fig. 3b). The MTS assay results showed that cell proliferation was also slowed in the ZNF384-targeted knockout group compared with that in the control group (Fig. 3c). Similarly, we analyzed the impact of ZNF384 protein knockout on the cell cycle via EdU immunofluorescence and flow cytometry experiments; these results also indicated cell cycle arrest at the G1/S phase transition when ZNF384 was knocked out (Fig. 3d, e).

Furthermore, we restored ZNF384 protein expression in ZNF384 knockout Huh7 cells (Fig. 4a). We found that the proliferative capacity of cells with restored ZNF384 expression was greater than that of knockout cells (Fig. $4 \mathrm{~b}$ ) and that the proportion of G1/S-phase cells could be restored to a level similar to that in the control groups (Fig. 4d). 


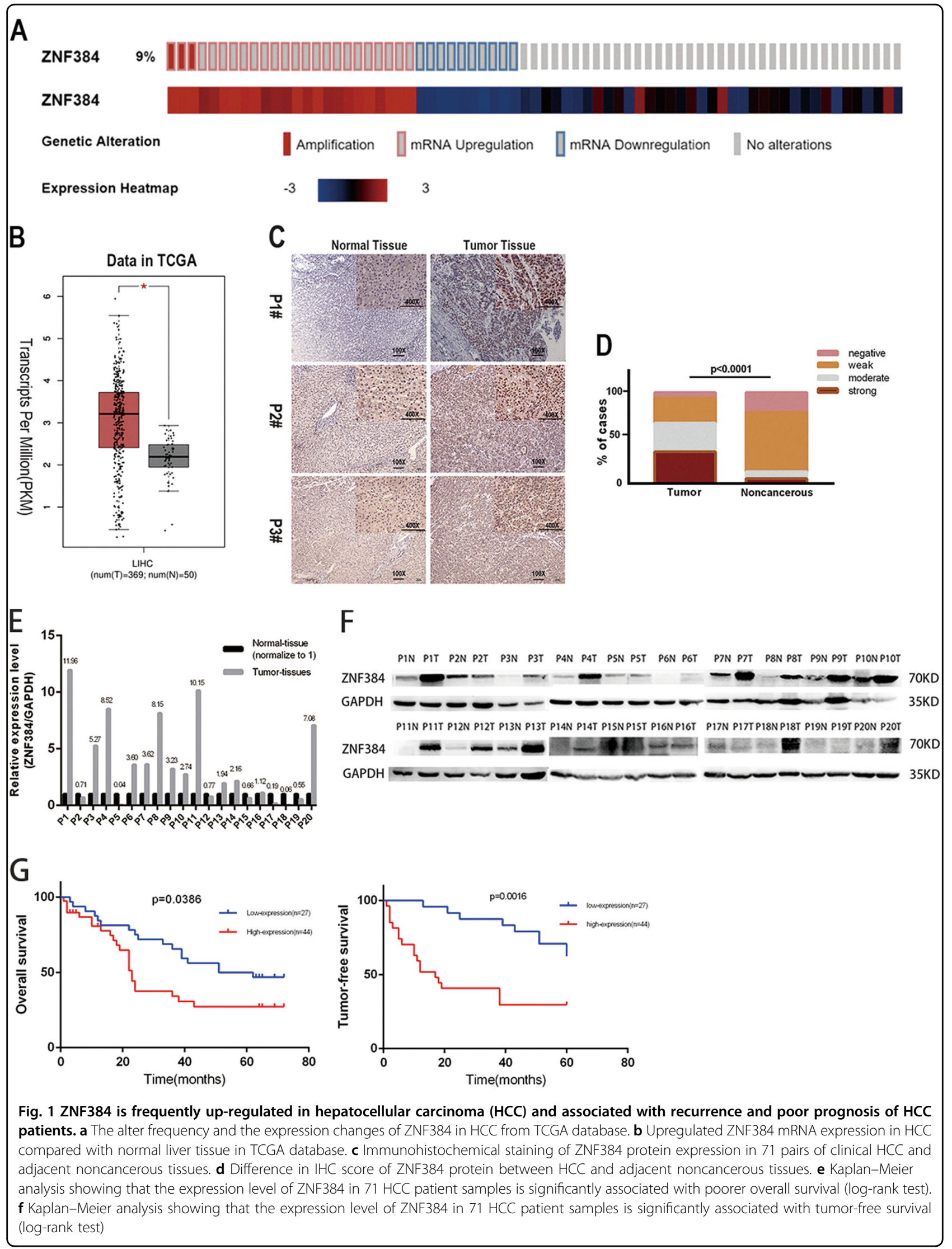


Table 1 Correlations between ZNF384 expression and clinicopathologic characteristics in 71 HCC patients

\begin{tabular}{|c|c|c|c|c|}
\hline & \multirow[t]{2}{*}{ Cases } & \multicolumn{2}{|c|}{ ZNF384 expression } & \multirow[t]{2}{*}{$P$-value } \\
\hline & & Low (\%) & High (\%) & \\
\hline Total & 71 & 38.03 & 61.97 & \\
\hline \multicolumn{5}{|l|}{ Age } \\
\hline$<60$ & 31 & 18.31 & 25.35 & \multirow[t]{2}{*}{0.5228} \\
\hline$\geq 60$ & 40 & 19.72 & 36.62 & \\
\hline \multicolumn{5}{|l|}{ Gender } \\
\hline Male & 58 & 33.80 & 47.89 & \multirow[t]{2}{*}{0.2250} \\
\hline Female & 13 & 4.23 & 14.08 & \\
\hline \multicolumn{5}{|c|}{$\operatorname{AFP}(n g / m l)$} \\
\hline$<400$ & 53 & 29.58 & 45.07 & \multirow[t]{2}{*}{0.6406} \\
\hline$\geq 400$ & 18 & 8.45 & 16.90 & \\
\hline \multicolumn{5}{|l|}{$\mathrm{HBsAg}$} \\
\hline Negative & 17 & 7.04 & 16.90 & \multirow[t]{2}{*}{0.4086} \\
\hline Positive & 54 & 30.99 & 45.07 & \\
\hline \multicolumn{5}{|l|}{ Size $(\mathrm{cm})$} \\
\hline$<5$ & 41 & 25.35 & 32.39 & \multirow[t]{2}{*}{0.2393} \\
\hline$\geq 5$ & 30 & 12.68 & 29.58 & \\
\hline \multicolumn{5}{|l|}{ Recurrence } \\
\hline No & 31 & 23.94 & 19.72 & \multirow[t]{2}{*}{0.0097} \\
\hline Yes & 40 & 14.08 & 42.25 & \\
\hline \multicolumn{5}{|c|}{ Liver cirrhosis } \\
\hline No & 34 & 18.31 & 29.58 & \multirow[t]{2}{*}{0.9730} \\
\hline Yes & 37 & 19.72 & 32.39 & \\
\hline
\end{tabular}

\section{ZNF384 promotes tumor growth in vivo}

An in vivo tumourigenesis assay was performed by subcutaneous injection of sgRNA1-Huh7 cells and control Huh7 cells into the left and right axillae of nude mice, respectively (Fig. 5a). Xenograft tumours were harvested after four weeks, and sgRNA1-Huh7 cells developed smaller tumours than control cells $(P=0.0094$, Fig. 5b, c).

\section{ZNF384 influence the cell cycle by regulating the expression of Cyclin D1}

To elucidate the mechanism of G1-to-S phase arrest caused by ZNF384 protein downregulation, we examined the expression changes of proteins involved in the G1/S phase transition, including Cyclin D1, p21, p27, CDK2, and CDK6, by western blotting. The results showed that the expression of Cyclin D1 was significantly reduced in ZNF384 knockdown HCCLM3 and PLC/PRF/5 cells. The same results were observed in ZNF384 knockout Huh7 cells. When we restored ZNF384 expression in these knockdown and knockout cells, we found that the expression of Cyclin D1 was restored (Fig. 6b). Moreover, we detected a significant positive correlation between the expression of ZNF384 and cyclin D1 in $60 \mathrm{HCC}$ samples $(P<0.0001$, Fig. 6c, d).

\section{ZNF384 recognizes the promoter region of CCND1 and regulates its transcription}

Previous results suggest a regulatory relationship between ZNF384 and Cyclin D1. Considering that ZNF384 is regarded to be a transcription factor, we speculated whether ZNF384 might directly regulate the transcription of Cyclin D1. Interestingly, we found through online analysis tools (http://jaspardev.genereg. net) that there might be ZNF384 recognition binding sites in the Cyclin D1 promoter region. Then, we used a luciferase reporter system and found that co-transfection of the ZNF384 plasmid and the wPGL3 plasmid caused a significant increase in luciferase units compared to that in the control groups (Fig. 6f). However, when we cotransfected the ZNF384 plasmid and mPGL3 plasmid, we did not observe significantly enhanced luciferase activity compared with that in the control groups (Fig. 6f). Thus, we performed ChIP analysis with cells overexpressing ZNF384-Flag. The results confirmed that ZNF384 is involved in the transcriptional regulation of Cyclin D1.

\section{Discussion}

In recent years, with the development of gene sequencing technology, genomic analyses show promise for improving tumor characterization and helping us develop individual treatment strategies for $\mathrm{HCC}$ patients ${ }^{14}$. A better understanding of the genetic alterations in HCC could contribute to identifying potential driver mutations and discovering novel therapeutic targets in the future $^{15,16}$. In this study, we showed that ZNF384, a gene with a genetic alteration rate of $9.4 \%$ in $\mathrm{HCC}$, was overexpressed in $\mathrm{HCC}$ and that the expression level of ZNF384 was associated with the prognosis of patients. When we downregulated the expression of ZNF384 via shRNA or CRISPR/Cas9 in HCC cell lines, cell proliferation was slowed both in vitro and in vivo. Cell cycle arrest at the G1/S transition was observed in ZNF384 knockdown cells. Finally, we verified that ZNF384 binds to the promoter region of Cyclin D1 and regulates the expression of Cyclin D1.

ZNF384 is a highly conserved gene expressed in a variety of human tissues ${ }^{17}$. This pattern of widespread expression suggests that ZNF384 has essential, evolutionarily conserved biological functions. Given that $\mathrm{HCC}$ is a highly heterogeneous cancer, there are significant differences in the oncogene and tumor suppressor gene mutation maps and the gene expression profiles among 


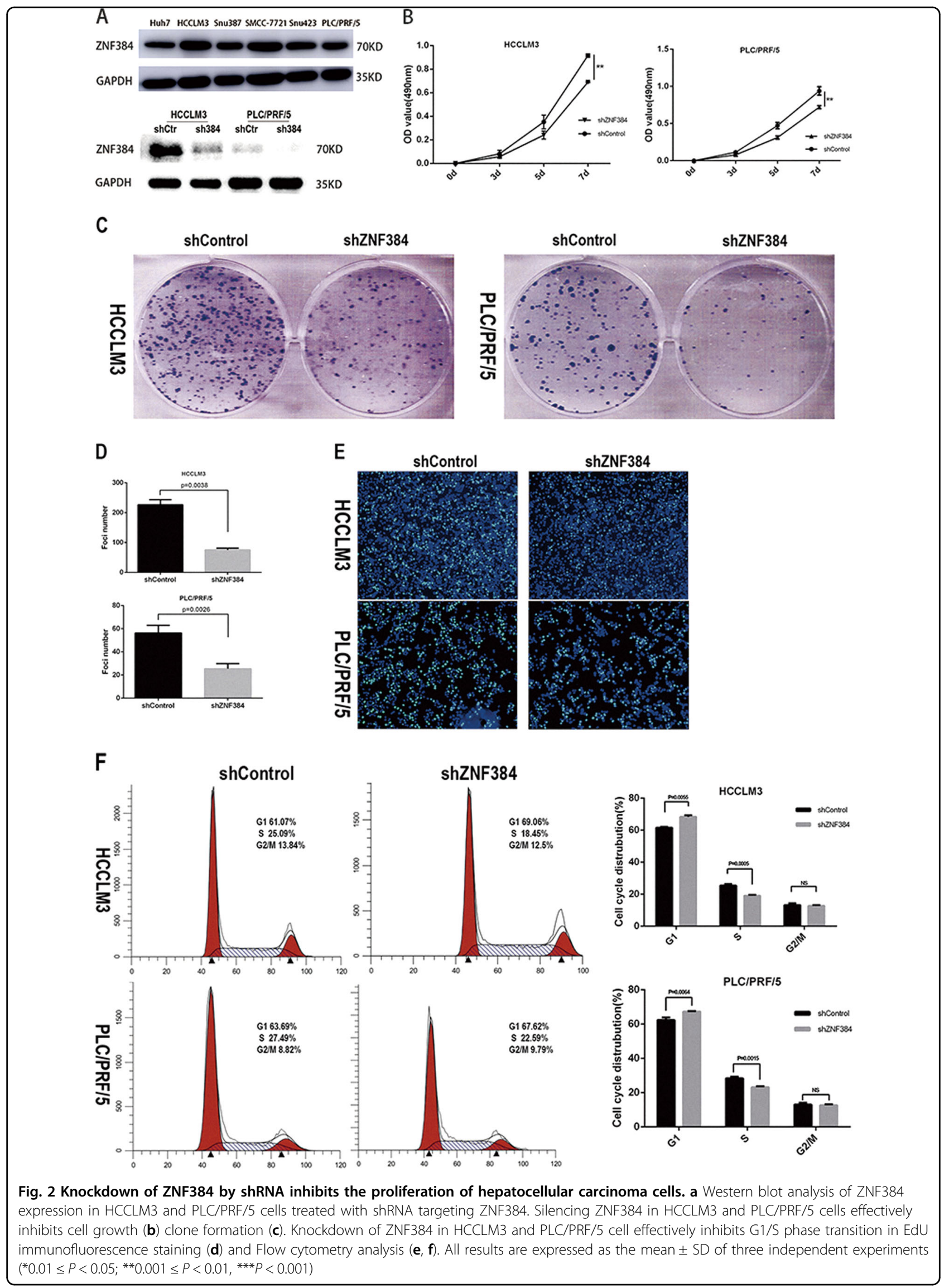


A

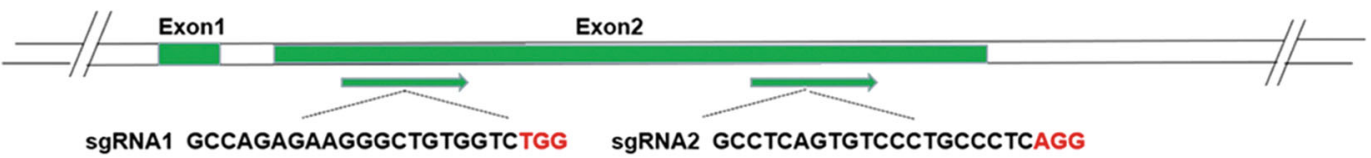

B
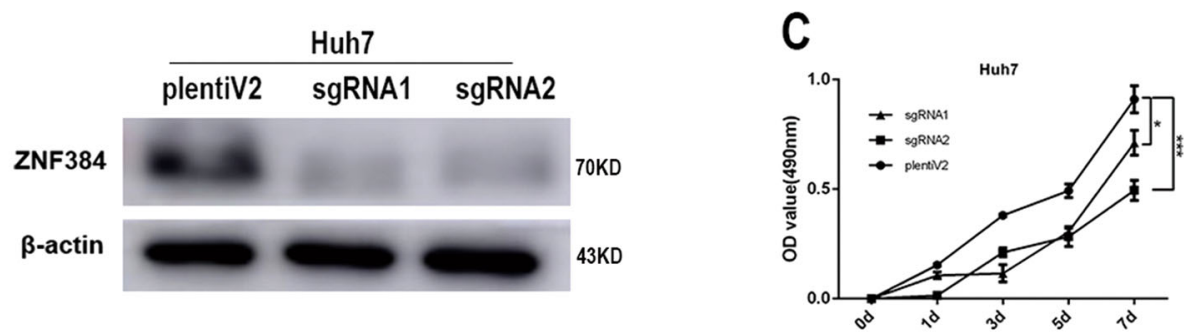

D
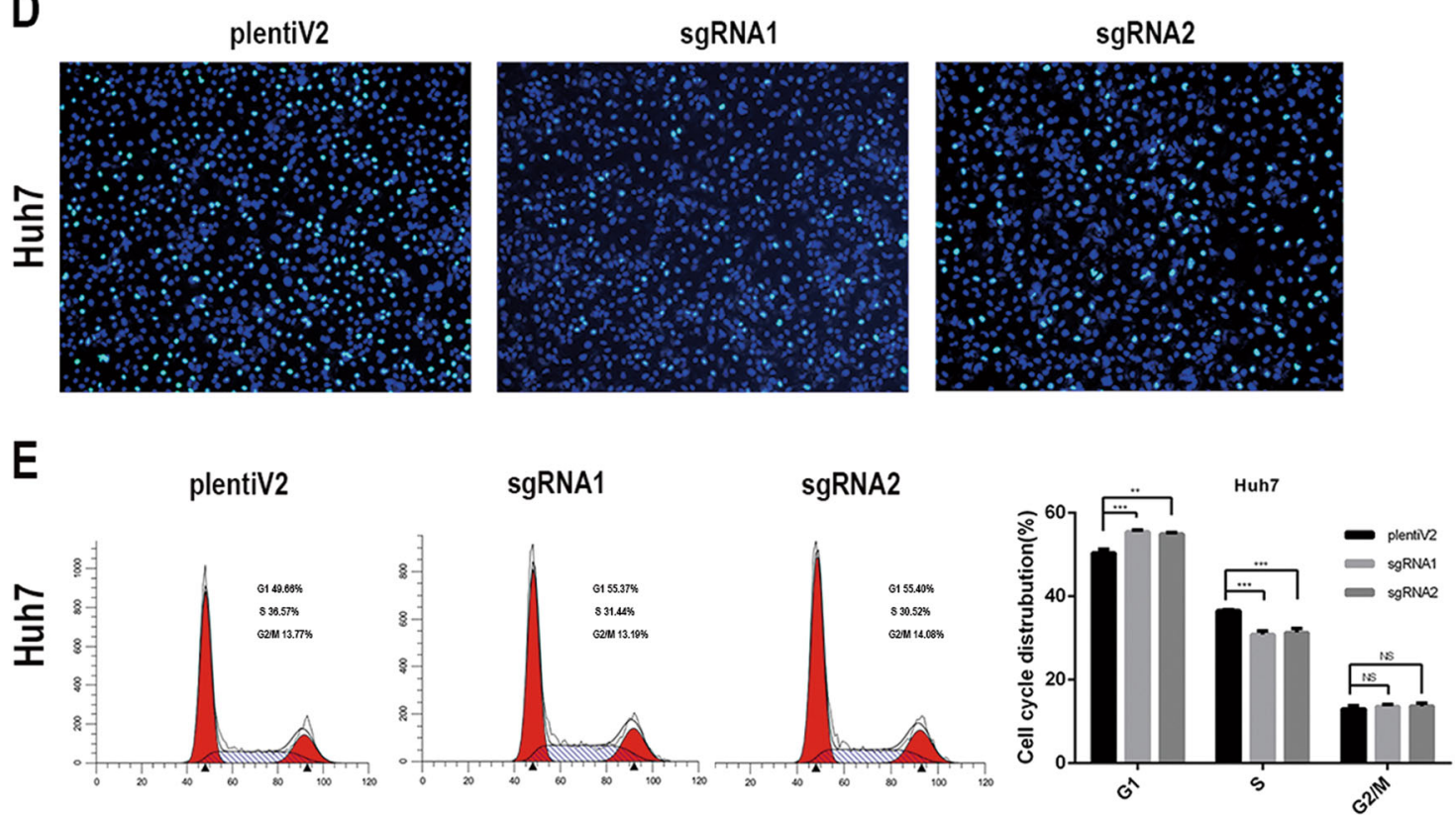

Fig. 3 Verify the effect of ZNF384 on the proliferation ability of hepatocellular carcinoma cells by knocking-out ZNF384 through Crispr/ CAS9. a The detail information of the two sgRNAs we chose. b Western blot analysis of ZNF384 expression in Huh7 cells treated with Crispr/CAS9 targeting ZNF384. c Knocking-out ZNF384 expression in Huh7 cells effectively inhibits cell growth. e, f Knocking-out ZNF384 expression in Huh7 cells effectively inhibits G1/S phase transition through Flow cytometry analysis. All results are expressed as the mean \pm SD of three independent experiments $\left({ }^{*} 0.01 \leq P<0.05 ;{ }^{* *} 0.001 \leq P<0.01,{ }^{* * *} P<0.001\right)$

liver cancer patients. It is worth noting that the tumor tissues of over $90 \%$ of the HCC patients in our study exhibited increased ZNF384 expression. It seems that ZNF384 plays a role as an oncogene in HCC. In a link to carcinogenesis, the recurrent rearrangement of ZNF384 with RNA-binding protein-coding genes such as EWSR1 or TAF15 have been observed and identified as oncogenic subtypes in acute leukemia $(\mathrm{AL})^{18}$. Additionally, the transactivating properties of the fusion protein were found in NIH3T3 cells, which implied the oncogenic potential of ZNF384 as a fusion protein ${ }^{19}$. Furthermore, it is reported that overexpression of ZNF384 can promote metastasis in melanoma cells ${ }^{12}$. Therefore, it is reasonable to speculate that HCC cells increase their ability to survive by expressing high levels of ZNF384 through unknown mechanisms. More studies are required to explore the molecular details underlying the regulation of ZNF384 expression in HCC.

The cell cycle is a series of precisely regulated steps orchestrated by specific cyclins that act in association with cyclin-dependent kinases $(\mathrm{CDKs})^{20}$. In normal cells, the cell cycle is precisely regulated. However, the ability to sustain unscheduled proliferation is a notable hallmark of cancer ${ }^{21}$. In this study, we found that reduced expression of ZNF384 blocked HCC cells from progressing from G1 phase to $\mathrm{S}$ phase with the downregulation of Cyclin D1 

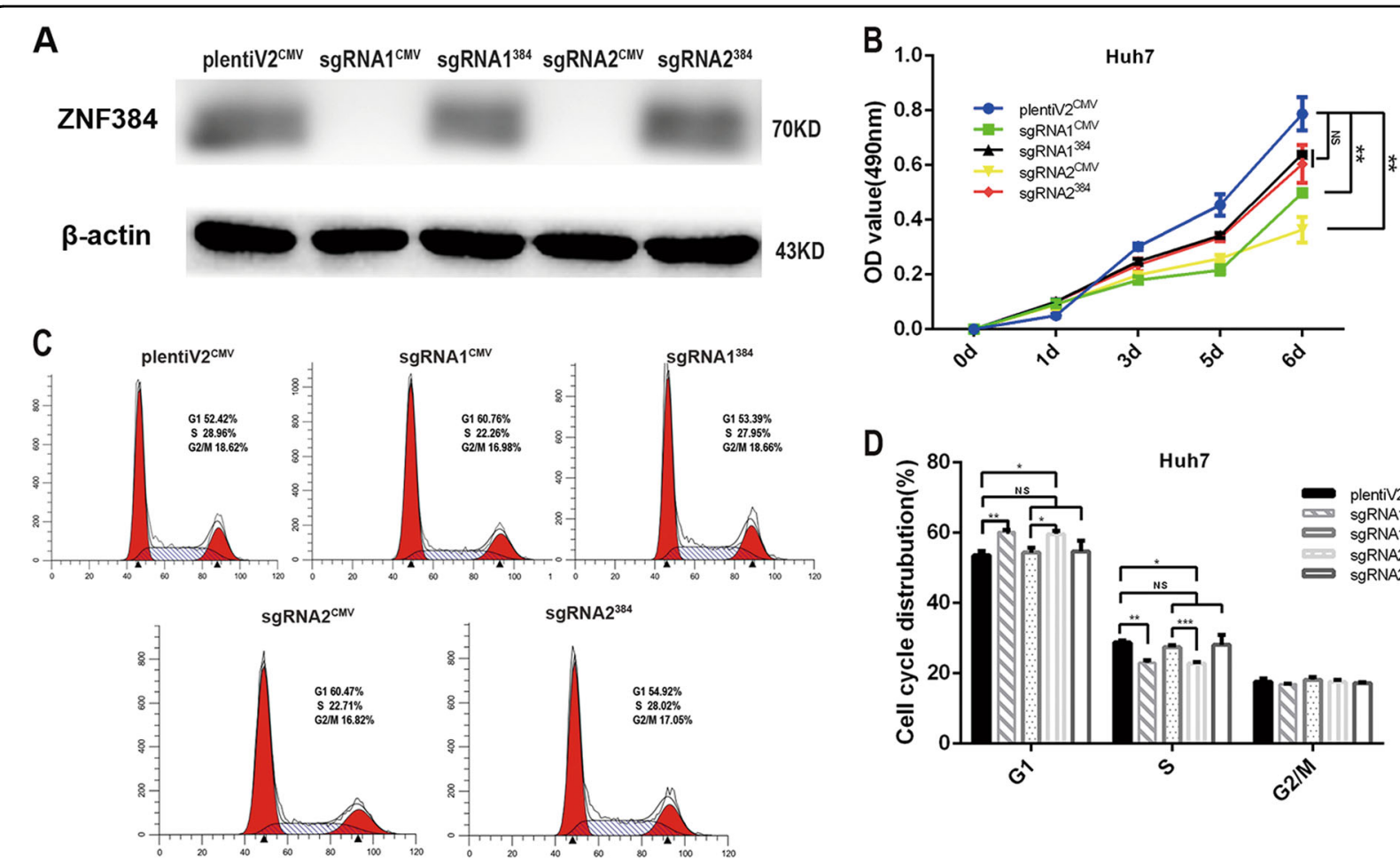

D

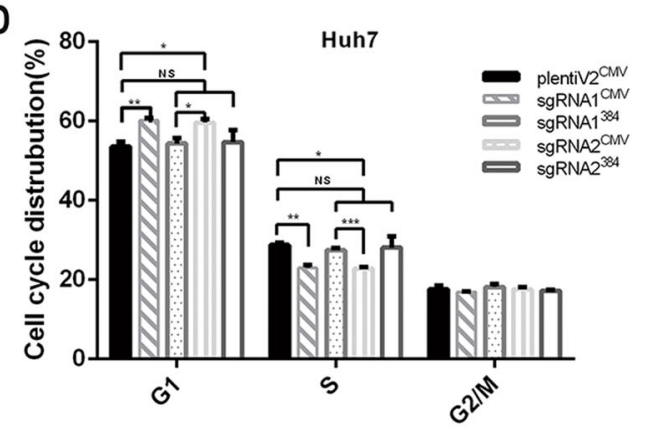

Fig. 4 Restoring ZNF384 protein in sgRNA-transfected Huh7 cells. a Western blotting shows that the expression of ZNF384 protein restored in sgRNA-Huh7 cells. b Restoring ZNF384 protein could reinstate the ability of cell proliferation. $\mathbf{c}$, d Restoring ZNF384 protein could eliminate the suppression of $\mathrm{G} 1 / \mathrm{S}$ phase transition

expression. Cyclin D1 is a protein encoded by the CCND1 gene, and it belongs to the highly conserved cyclin family, whose members are characterized by periodic changes in protein expression throughout the cell cycle $^{22}$. The function of cyclin D1 in cell cycle control is believed to be mediated through interaction with CDK4 and/or CDK6 to promote G1/S phase transition ${ }^{23,24}$. Therefore, overexpression of Cyclin D1 is frequently observed to promote cancer cell proliferation in a variety of cancers, including HCC. The relationship between ZNF384 and Cyclin D1 was further confirmed in clinical samples by IHC. In addition, it is reported that advanced-stage HCCs potentially harbor more Cyclin D1 amplifications, and the amplification of Cyclin D1 is also associated with poor prognosis in $\mathrm{HCC}^{25}$. This finding is consistent with our finding in this study that high ZNF384 expression often predicts a worse prognosis for patients.

ZNF384 is a transcription factor containing a $\mathrm{C} 2 \mathrm{H} 2$ zinc finger structure, and previous studies have shown that it regulates MMP1, MMP3, and COL1A1 gene transcription $^{26-28}$. It is predicted that the transcriptional recognition site is a sequence with adenine enrichment. Moreover, we found an adenine-enriched sequence in the promoter region of Cyclin D1. Thus, we postulated that Cyclin D1 might also be a target gene of ZNF384, a hypothesis that was verified through a luciferase assay.
These results demonstrate that ZNF384 specifically targets the promoter region of Cyclin D1 and regulates the expression of Cyclin D1.

In summary, our study first described the role of ZNF384 as an oncogene in the development of HCC. ZNF384 promotes the proliferation of HCC cells by upregulating the expression of Cyclin D1. In addition, we provide evidence that ZNF384 directly regulates Cyclin D1 transcription in HCC. In the future, ZNF384 might serve as a prognostic or predictive factor and as a potential therapeutic target in HCC diagnosis and treatment.

\section{Materials and methods \\ Patients and specimens}

We collected 51 paired HCC samples and adjacent noncancerous tissues from patients who underwent liver resection in Sir Run Run Shaw Hospital (SRRSH) from January 2006 to December 2010 and obtained another 20 paired HCC samples and adjacent noncancerous tissues from patients who underwent liver resection in Sir Run Run Shaw Hospital (SRRSH) from May 2017 to September 2017. All specimens were immobilized in formalin immediately and further embedded for subsequent pathological testing. In addition, we also acquired 20 pairs of fresh frozen tissue specimens from the hospital tissue 


\section{A}

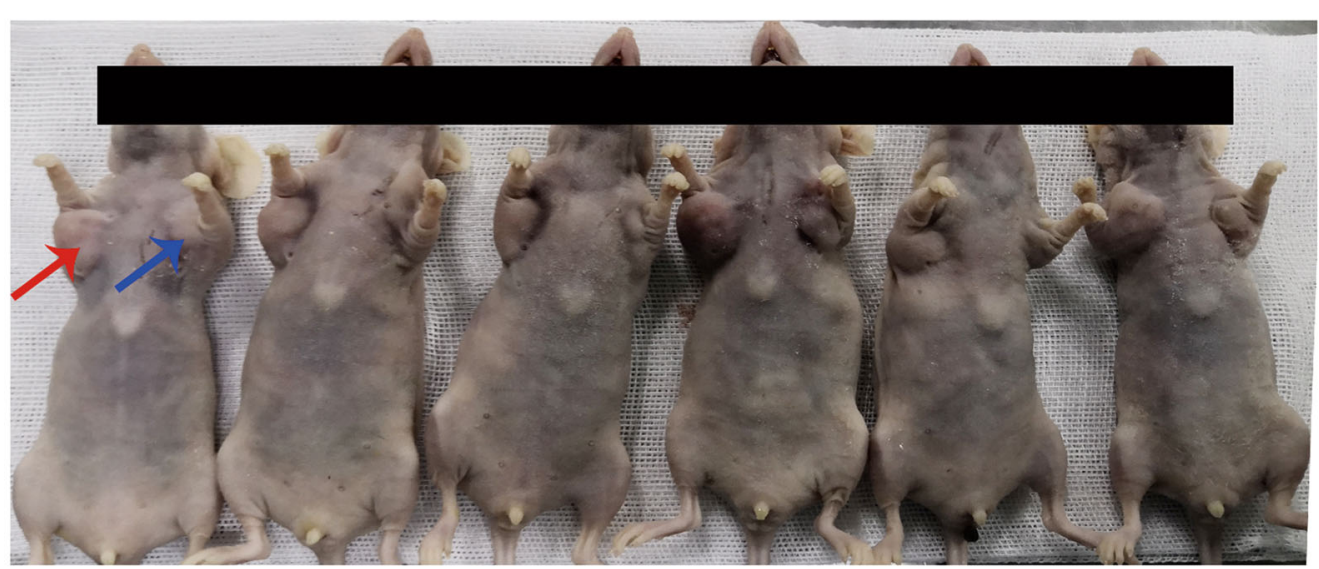

B

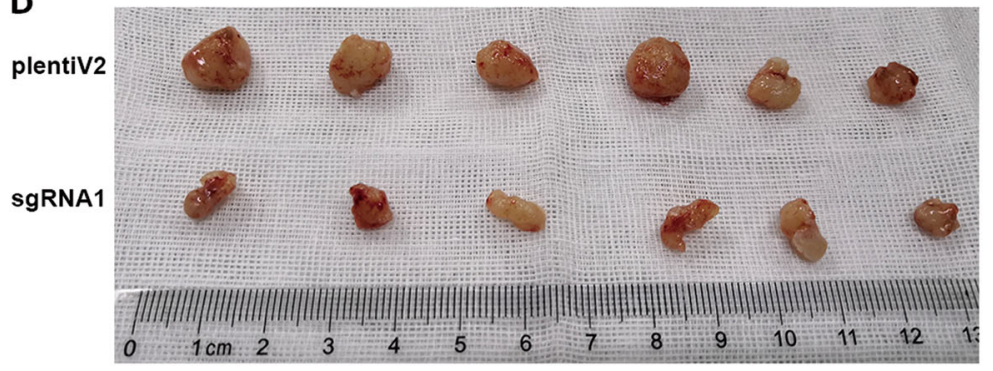

C

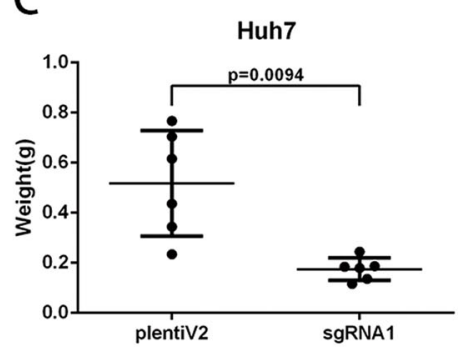

Fig. 5 ZNF384 promoter tumor growth in vivo. a Images of xenograft tumors formed in nude mice injected with sgRNA1-Transfected Huh7 cells (Left, red arrows) and empty vector-transfected Huh7 cells (Right, blue arrows). b Tumors formed in nude mice. c Tumor weights were analyzed by independent Student's $t$-test

bank for quantitative polymerase chain reaction (PCR) analysis and western blot analysis. Detailed information about the clinical and pathological characteristics is listed in Table 1. The American Joint Committee on Cancer (AJCC) staging system was used to evaluate tumor stages, and the Barcelona Clinic Liver Cancer (BCLC) staging system was used to define clinical stages. The study was approved by the Medical Ethics Committee of SRRSH, and all patients were informed.

\section{Quantitative real-time PCR analysis}

Total RNA was extracted from tissue samples using Trizol reagent (Thermo Fisher Scientific cat\#204211) according to the manufacturer's instructions. cDNA was synthesized from $1 \mu \mathrm{g}$ of RNA of each sample using an iScript cDNA synthesis kit (YEASEN, cat\#11121ES60). qRT-PCR was performed using a qPCR SYBR Green Master Mix (YEASEN, cat\#11198ES08). The ZNF384 primer sequences were as follows: forward, 5'GTCTCAGGTCAGATCGAGAACA-3' and reverse $5^{\prime}$ ACTCTGTGTCCATACTGATGCC- $3^{\prime}$. The GAPDH primer sequences were as follows: forward, $5^{\prime}$-GT GAAGCAGGCGTCGGA' and reverse, 5'-AGCCC CAGCGTCAAAGG-3'. Each sample was tested in triplicate. Data were analyzed using the $2^{-\Delta \Delta C T}$ calculation method.

\section{Cell culture}

The HCC cell lines Huh7, HCCLM3, Snu423, Snu387, SMCC-7721, and PLC/PRF/5 were kindly provided by the Cang laboratory at Zhejiang University. Huh7, HCCLM3, and PLC/PRF/5 cells were cultured in DMEM (Gibco, cat\#C11995500BT) supplemented with 10\% fetal bovine serum (FBS) (Cellmax, cat\#SA102.02). Snu423, Snu387, and SMCC-7721 cells were cultured in RPMI-1640 (Gibco, cat\# C11975500BT) supplemented with 10\% FBS (Cellmax, cat\#SA102.02). The culture environment was maintained at $37^{\circ} \mathrm{C}$ with $5 \% \mathrm{CO}_{2}$.

\section{Plasmid constructs}

First, we obtained the sequence for shRNA targeting ZNF384 from http://www.sigmaaldrich.com. The shRNA sequence was as follows: forward, 5'-CCGGCCCGAGATGAATGACCCTTATTTCAAGAGAATAAGGGTC ATTCATCTCGGGTTTTTTGGTACC-3' and reverse, 5'-AATTGGTACCAAAAAACCCGAGATGAATGACC CTTATAAGTTCTCTATAAGGGTCATTCATCTCGG G-3'. Then, we cloned the shRNA into the pLKO.1 puro 

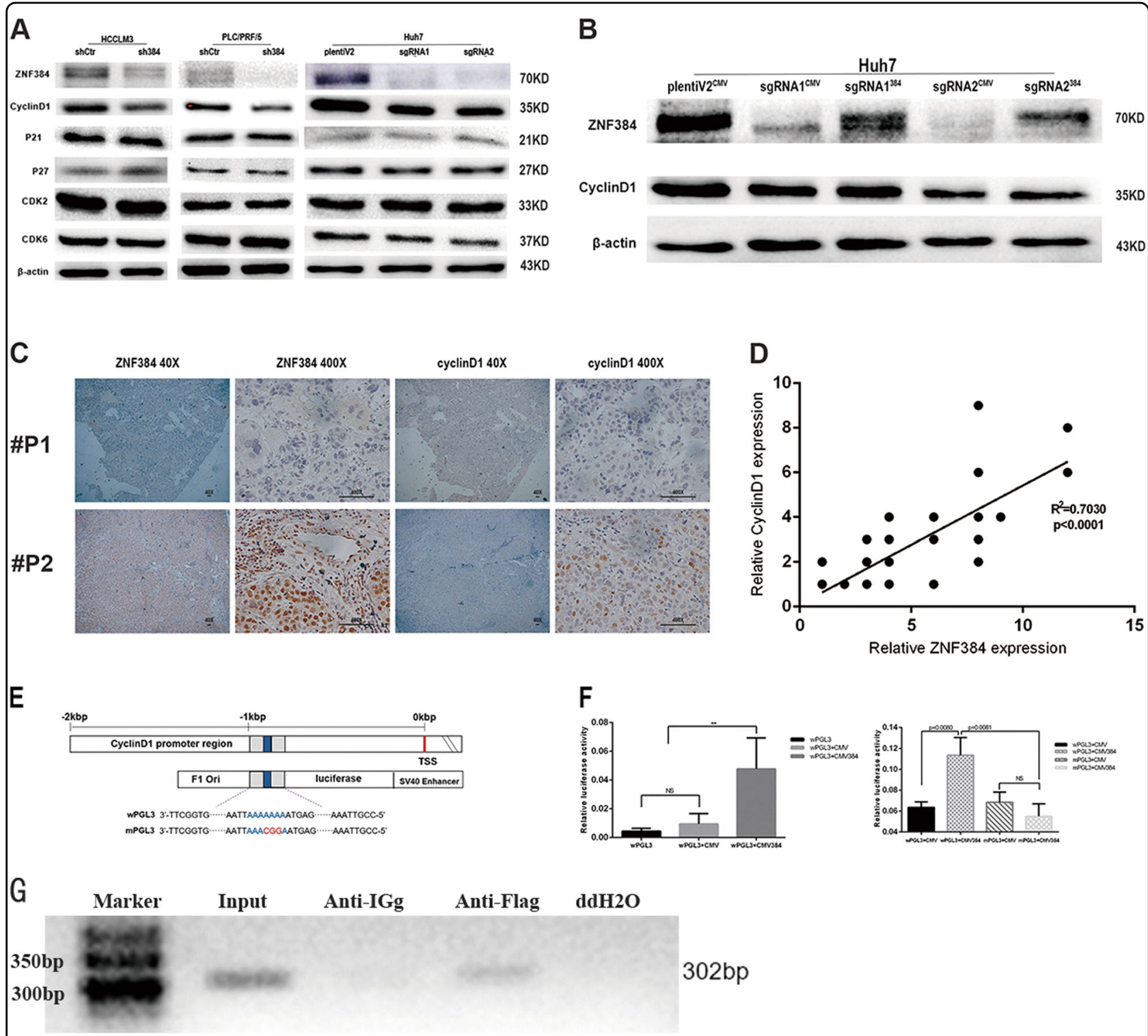

Fig. 6 ZNF384 binds to the promoter region of cyclinD1 to regulate the expression of cyclin D1. a Western blotting shows that the expression of Cyclin D1 was down-regulated in both of shZNF384-transfected and sgRNA-transfected cells compared with empty vector-transfected cells. b Western blotting shows that the expression of Cyclin D1 recovered when we restored ZNF384 expression in sgRNA-transfected cells. c The expression of ZNF384 and Cyclin D1 in 60 HCC samples through IHC. $\mathbf{d}$ The correlation between the expression of ZNF384 and Cyclin D1 in correlation analysis ( $n=60$, part of dots was overlapped). e The structure of the luciferase plasmid in this study. $\mathbf{f}$ ZNF384 recognizes and bound to the promoter region of Cyclin D1 and regulates Cyclin D1 transcription. $\mathbf{g}$ Binding of ZNF384-Flag to the predicted combining capacity in Cyclin D1 promoter region by ChIP assays. Input DNA was used as positive control and $\mathrm{ddH}_{2} \mathrm{O}$ was used as a negative control

plasmid (Sigma, cat\#SHC001) at the AgeI and EcoRI restriction sites according to the manufacturer's instructions. We also designed the single guide RNA (sgRNA) using the online sgRNA design website (http://crispr.mit. edu). The sgRNA was cloned into the lentiCRISPRv2 plasmid (Addgene, cat\#52961) according to the established protocol. Full-length ZNF384 cDNA was generously provided by the Jiahuai Han laboratory (Xiamen
University). The cDNA was amplified and cloned into the p3xFlag-CMV-7.1 expression vector (Sigma, cat\#E4026) using XbaI and BamHI. Finally, we amplified the fragment located at the promoter region of Cyclin D1. Subsequently, this fragment was PCR amplified and cloned upstream of the firefly luciferase gene in the pGL3Enhancer vector (Promega, cat\#E1771) using NheI and HindIII. This plasmid was termed wPGL3. Using a 
Table 2 Detail information for the primers we used. F, forward; R: reverse

\begin{tabular}{ll}
\hline Name & Sequences $\left(\mathbf{5}^{\prime} \mathbf{3}^{\prime}\right.$ ) \\
\hline sgRNA-1 & F: CACCGGCCTCAGTGTCCCTGCCCTC \\
sgRNA-1 & R: AAACGAGGGCAGGGACACTGAGGCC \\
sgRNA-2 & F: CACCGGCCAGAGAAGGGCTGTGGTC \\
sgRNA-2 & R: AAACGACCACAGCCCTTCTCTGGCC \\
Xbal-ZNF384 & F: GCCTCTAGAATGGAAGAATCTCACTTCA \\
BamHI-ZNF384 & R: TTTGATCCCTAAGAGCTGGCCAGGTGC \\
Nhel-CCND1 & F: CTAGCTAGCCACCCCCAACAAAACCAATT \\
HindIII-CCND1 & R: CCCAAGCTITCCTACCTTGACCAGTCGG \\
Mutate-CCND1 & F: ATTTCTITITAATTAAACGGAATGAGTCAG \\
Mutate-CCND1 & R: GATCTCCATTCTGACTCATTCCGTTTAATTA \\
\hline
\end{tabular}

Mut Express Multis Fast Mutagenesis Kit (Vazyme, cat\#C215-01), we performed site-directed mutagenesis of the ZNF384 binding sites in the Cyclin D1 promoter region. This plasmid was termed mPGL3. Detailed information for the primers we used is provided in Table 2.

\section{Lentivirus production and transduction}

Lentivirus expressing shRNA was produced by cotransfecting HEK293T cells with a mixture of shZNF384 or shControl plasmid, the Gag-Pol packaging plasmid psPAX2 (Addgene, cat\#12260), and the envelope plasmid pMD2G (Addgene, cat\#12259) at a 4:3:2 ratio using Lipofectamine 2000 transfection (lip2000) reagent (Thermo Fisher, cat\#11668019). Lentivirus expressing sgRNA and cas9 was produced by co-transfecting HEK293T cells with a cocktail of gRNA/Cas9-expressing lentiCRISPRv2, Gag-Pol packaging plasmids, including psPAX2 (Addgene, cat\#12260), and B19/VSVG (Addgene, cat\#88865) at a ratio of 3:4:1 using lip2000 reagent. Virus particles were harvested $48 \mathrm{~h}$ after transfection. Lentivirus-infected cells were screened with the corresponding concentration of puromycin (Thermo Fisher, cat\#A1113802: Huh7, $1 \mu \mathrm{g} / \mu \mathrm{l}$; PLC/ PRF $/ 5,1 \mu \mathrm{g} / \mu \mathrm{l}$; HCCLM3, $10 \mu \mathrm{g} / \mu \mathrm{l})$.

\section{MTS cell proliferation assay and colony formation assay}

For the cell growth assay, we seeded $1 \times 10^{3}$ cells per well into 96-well plates. After the cells were attached, we added $5 \mathrm{mg} / \mathrm{ml}$ MTS (Promega, cat\#G3580) to each well at $0 \mathrm{~h}$ for 1 day, 3 days, 5 days, and 7 days, and the absorbance values were measured at a wavelength of $490 \mathrm{~nm}$ by spectrophotometry.

In the colony formation assay, $1 \times 10^{3}$ cells were seeded in 6-well culture plates. Two weeks later, the cells were fixed in $4 \%$ paraformaldehyde, stained with crystal violet solution (Beyotime Biotechnology, cat\#C0121) and counted under a microscope.

\section{EdU incorporation assay}

ZNF384 knockdown or knockout HCC cells and their corresponding control cells were seeded in a 24-well culture plate at $2 \times 10^{5}$ cells/well and incubated for $24 \mathrm{~h}$. A 5-ethynyl-20-deoxyuridine (EdU) assay using an EdU assay kit (Beyotime Biotechnology, cat\#C0071S) was used to assess cell proliferation according to the manufacturer's protocol. First, cells were incubated with $10 \mu \mathrm{M}$ EdU for $3 \mathrm{~h}$ at $37^{\circ} \mathrm{C}$ and were then fixed with $4 \%$ paraformaldehyde. After the samples were treated with $0.3 \%$ Triton X100 for $10 \mathrm{~min}$, the cells were stained with Azide 488 for $30 \mathrm{~min}$. Subsequently, cell nuclei were stained with Hoechst 33342 for $10 \mathrm{~min}$. Samples were analyzed by a fluorescence microscope (Olympus).

\section{Flow cytometry analysis}

Cell cycle distribution was analyzed by flow cytometry (BD LSRFortessa ${ }^{\mathrm{TM}} \mathrm{X}-20$ ). Cells were treated with cell cycle staining buffer (MultiSciences, cat\#CCS01) according to the manufacturer's instructions.

\section{Western blotting and antibodies}

Cells were harvested and lysed in radioimmunoprecipitation assay buffer (1\% NP40, $0.5 \%$ sodium deoxycholate, $0.1 \%$ sodium dodecyl sulfate, $0.03 \%$ aprotinin, $10 \mathrm{ng} / \mathrm{ml}$ phenylmethylsulfonyl fluoride, and $1 \mu \mathrm{M}$ sodium orthovanadate) at $4{ }^{\circ} \mathrm{C}$ for $30 \mathrm{~min}$. The protein concentration was quantified using a BCA protein assay kit (Thermo Fisher Scientific, cat\#A53227). Proteins were separated via $10 \%$ SDS-polyacrylamide gel electrophoresis (SDS-PAGE). Next, samples were transferred to $0.22 \mu \mathrm{m}$ thick polyvinylidene difluoride (PVDF) membranes (Merck Millipore, Billerica, MA, USA). Non-specific binding sites on the membranes were blocked for $1 \mathrm{~h}$ with 5\% non-fat milk (BD, cat\#232100). After blocking, membranes were incubated first with a primary antibody and then with a secondary antibody. Finally, immunoreactions were visualized using Clarity Western ECL substrate (Bio-Rad, cat\#VL001) and the blots were imaged using a luminescent image analyser (Fujifilm, Tokyo, Japan). The following antibodies were used: anti-ZNF384 (Atlas Antibodies, HPA004051), anti-Cdk2 (Abcam, cat\#ab32147), anti-Cdk6 (Abcam, cat\#ab124821), antiCyclin D1 (Abcam, cat\#ab134175), anti-p21 (Abcam, cat\#ab109199), anti-p27 (Abcam, cat\#ab32034), antiDDDDK (Abcam, cat\#ab1162), and anti-GAPDH (Abcam, cat\#ab181602).

\section{Immunohistochemical staining (IHC)}

IHC was used to evaluate the expression of ZNF384 in HCC tissues and paired noncancerous tissues. Paraffin- 
embedded sections were provided by the department of pathology. First, these sections were deparaffinized and rehydrated. For antigen retrieval, the sections were immersed in $10 \mathrm{mM}$ citrate buffer ( $\mathrm{pH}$ 6.0) and boiled for $10 \mathrm{~min}$ in a microwave oven. Then, endogenous peroxidase activity was blocked with $3 \%$ hydrogen peroxide for $10 \mathrm{~min}$. Non-specific binding sites were blocked with 5\% normal goat serum for $30 \mathrm{~min}$. The sections were incubated with an antibody against ZNF384 (1:100, Atlas Antibodies, cat\#HPA004051) overnight at $4{ }^{\circ} \mathrm{C}$. The sections were then incubated with the secondary antibody, and the expression of ZNF384 in the tissues was observed via microscopy after $D A B$ staining and haematoxylin staining. The score was evaluated by two pathologists blinded to the clinicopathological data using a German immunoreactivity score ${ }^{29}$.

\section{In vivo tumorigenicity assay}

A xenograft mouse model was used to verify the oncogenic function of ZNF384 in vivo. BALB/c nude male mice (4 weeks of age) were subcutaneously injected with Huh7/sgRNA1 knockout cells $\left(1 \times 10^{6}\right.$, subcutaneous) or Huh7/lentiV2 control cells $\left(1 \times 10^{6}\right.$, subcutaneous $)$ in $150 \mu \mathrm{l}$ of PBS. Mice were sacrificed after four weeks, and the tumours were harvested and measured. The BALB/c nude mice ( $n=6$, male, 4 weeks of age) were obtained from Shanghai Laboratory Animal Center and housed in the laboratory animal research center of SRRSH. All animal experiments were conducted with consent from the Committee of the Use of Live Animals in Teaching and Research at Sir Run Run Shaw Hospital.

\section{Dual-luciferase reporter assay}

HEK293T cells were separately transfected with the vectors wPGL3 or mPGL3. ZNF384 was overexpressed by the addition of the ZNF384 expression vector (pMSCVFlag-hSOX2, Addgene, cat\#2007). Luciferase activity was normalized to that of cells co-transfected with the pRL Renilla expression vector (Promega, cat\#E2231). Lysates were analyzed with a dual luciferase kit (Promega, cat\#E1980).

\section{Chromatin immunoprecipitation assay}

LM3 cells were transfected with the CMV-Flag plasmid for 3 days and cross-linked with $1 \%$ formaldehyde for $10 \mathrm{~min}$ at $37^{\circ} \mathrm{C}$ in $10 \mathrm{ml}$ of DMEM. Then, we added $1.1 \mathrm{ml}$ of glycine solution $(10 \times)$ to the reactions at room temperature. After $5 \mathrm{~min}$, cells were washed three times with PBS containing $1 \mathrm{mM}$ PMSF and resuspended in $200 \mu \mathrm{l}$ of lysis buffer containing $1 \mathrm{mM}$ PMSF. Subsequently, cell lysates were treated with a Misonix Sonicator 3000 Homogenizer (Mandel Scientific Company Inc., Guelph, ON, Canada) to fragment genomic DNA into sizes of $200-800 \mathrm{bp}$. After the samples were centrifuged at $4{ }^{\circ} \mathrm{C}$ and $12,000 \times g$ for $5 \mathrm{~min}$, we removed the supernatant and added $1.8 \mathrm{ml}$ of ChIP Dilution Buffer containing $1 \mathrm{mM}$ PMSF to the supernatant. Twenty microliters of each sample were removed and used as input for subsequent detection. The remaining sample volumes were precleared with $70 \mu \mathrm{l}$ of protein $\mathrm{A} / \mathrm{G}$ agarose for $30 \mathrm{~min}$ at $4{ }^{\circ} \mathrm{C}$. After removing the protein $\mathrm{A} / \mathrm{G}$ agarose, anti-DDDK flag and anti-IgG antibodies were used to immunoprecipitate the samples on a shaker at $4{ }^{\circ} \mathrm{C}$ overnight. After centrifugation, the supernatant was discarded, and the protein A/G agarose was washed successively in Low Salt Immune Complex Wash Buffer, High Salt Immune Complex Wash Buffer, LiCl Immune Complex Wash Buffer and TE Buffer. Finally, DNA was eluted from the protein A/G agarose with elution buffer, and $20 \mu \mathrm{l}$ of $5 \mathrm{M}$ $\mathrm{NaCl}$ was added to the solution to eliminate cross-linking between proteins and genomic DNA. The DNA was analyzed via PCR with the following primers: forward primer, $5^{\prime}$-tctaaaggtgaagggacgtc- $3^{\prime}$ and reverse primer, $5^{\prime}$ ccttcatcttgtccttctagc- $3^{\prime}$.

\section{Statistical analysis}

All results are presented as the means \pm SDs. The differences between categorical variables were assessed by the $\chi^{2}$ test, and the differences between two groups were assessed by Student's $t$-test. HCC survival data such as OS and DFS were analyzed with Kaplan-Meier curves and the log-rank test. Statistical analysis was performed using SPSS 22.0 software (SPSS Inc., Chicago, IL, USA). A Pvalue of $<0.05$ was considered statistically significant.

\section{Acknowledgements \\ We are greatly indebted to the subjects enrolled in our study. This work was supported by the National Key Research and Development Program (2016YFC0906400), National Natural Science Foundation of China (81872297, and 81874059), Zhejiang province analysis and test technology project (2018C37062), the Fundamental Research Funds for the Central Universities (2016XZZX002-05).}

\section{Author details \\ ${ }^{1}$ Department of General Surgery, Sir Run Run Shaw Hospital, School of Medicine, Zhejiang University, Hangzhou, China. 'Biomedical Research Center, Sir Run Run Shaw Hospital, School of Medicine, Zhejiang University, Hangzhou, China. ${ }^{3}$ School of Life Science and Technology, Nanyang Normal University, Nanyang, China. ${ }^{4}$ Key Laboratory of Arrhythmias of the Ministry of Education of China, East Hospital, Tongji University School of Medicine, Institute of Medical Genetics, Tongji University, Shanghai, China}

Conflict of interest

The authors declare that they have no conflict of interest.

\section{Publisher's note}

Springer Nature remains neutral with regard to jurisdictional claims in published maps and institutional affiliations.

Received: 20 January 2019 Revised: 15 May 2019 Accepted: 16 May 2019 Published online: 05 June 2019 


\section{References}

1. Lin, D. C. et al. Genomic and epigenomic heterogeneity of hepatocellular carcinoma. Cancer Res. 77, 2255-2265 (2017).

2. Fu, J. \& Wang, H. Precision diagnosis and treatment of liver cancer in China Cancer Lett. 412, 283-288 (2018).

3. Llovet, J. M. \& Bruix, J. Molecular targeted therapies in hepatocellular carcinoma. Hepatology 48, 1312-1327 (2008).

4. Gao, Q. et al. Cell culture system for analysis of genetic heterogeneity within hepatocellular carcinomas and response to pharmacologic agents. Gastroenterology 152, 232-242 (2017).

5. Niu, Z. S., Niu, X. J. \& Wang, W. H. Genetic alterations in hepatocellular carcinoma: an update. World J Gastroenterol 22, 9069-9095 (2016).

6. Sia, D., Villanueva, A., Friedman, S. L. \& Llovet, J. M. Liver cancer cell of origin molecular class, and effects on patient prognosis. Gastroenterology $\mathbf{1 5 2}$ 745-761 (2017)

7. Rimassa, L., Pressiani, T., Personeni, N. \& Santoro, A. Regorafenib for the treatment of unresectable hepatocellular carcinoma. Expert Rev Anticancer The 17, 567-576 (2017)

8. Llovet, J. M. et al. Sorafenib in advanced hepatocellular carcinoma. $N$ Engl J Med 359, 378-390 (2008).

9. Nakamoto, T. et al. ClZ, a zinc finger protein that interacts withp130(cas) and activates the expression of matrix metalloproteinases. Mol Cell Biol $\mathbf{2 0}$ 1649-1658 (2000)

10. Zhong, C. H. et al. E2A-ZNF384 and NOL1-E2A fusion created by a cryptic t (12;19)(p13.3; p13.3) in acute leukemia. Leukemia 22, 723-729 (2008).

11. Do Amaral, A. Complexities of nomenclature in biology. Gender of generic names ending in ops $z, n,(s)$ 1572]. Mem Inst Butantan 39, 27-36 (1975).

12. Sakuma, T. et al. CIZ/NMP4 is expressed in B16 melanoma and forms a positive feedback loop with RANKL to promote migration of the melanoma cells. J Cell Physiol 227, 2807-2812 (2012).

13. Mori, S., Takeuchi, T., Ishii, Y. \& Kukimoto, I. Identification of APOBEC3B promoter elements responsible for activation by human papillomavirus type 16 E6. Biochem Biophys Res Commun 460, 555-560 (2015).

14. Tang, $L$. et al. The prognostic significance and therapeutic potential of hedgehog signaling in intrahepatic cholangiocellular carcinoma. Clin Cancer Res 19, 2014-2024 (2013).
15. Schulze, K. et al. Exome sequencing of hepatocellular carcinomas identifies new mutational signatures and potential therapeutic targets. Nat Genet 47, 505-511 (2015)

16. Llovet, J. M. \& Hernandez-Gea, V. Hepatocellular carcinoma: reasons for phase III failure and novel perspectives on trial design. Clin Cancer Res 20, 2072-2079 (2014).

17. Bruix, J., Gores, G. J. \& Mazzaferro, V. Hepatocellular carcinoma: clinical frontiers and perspectives. Gut 63, 844-855 (2014).

18. Childress, P., Robling, A. G. \& Bidwell, J. P. Nmp4/ClZ: road block at the intersection of PTH and load. Bone 46, 259-266 (2010).

19. Lilljebjorn, H. \& Fioretos, T. New oncogenic subtypes in pediatric B-cell precursor acute lymphoblastic leukemia. Blood 130, 1395-1401 (2017).

20. Martini, A. et al. Recurrent rearrangement of the Ewing's sarcoma gene, EWSR1, or its homologue, TAF15, with the transcription factor CIZ/NMP4 in acute leukemia. Cancer Res 62, 5408-5412 (2002).

21. Malumbres, M. Cyclin-dependent kinases. Genome Bio/ 15, 122 (2014)

22. Ingham, M. \& Schwartz, G. K. Cell-cycle therapeutics come of age. J Clin Oncol 35, 2949-2959 (2017)

23. Kenny, F. S. et al. Overexpression of cyclin D1 messenger RNA predicts for poor prognosis in estrogen receptor-positive breast cancer. Clin Cancer Res $\mathbf{5}$ 2069-2076 (1999).

24. Knudsen, K. E., Diehl, J. A., Haiman, C. A. \& Knudsen, E. S. Cyclin D1: polymorphism, aberrant splicing and cancer risk. Oncogene 25, 1620-1628 (2006)

25. Baldin, V. Lukas, J., Marcote, M. J. Pagano, M. \& Draetta, G. Cyclin D1 is a nuclear protein required for cell cycle progression in G1. Genes Dev 7, 812-821 (1993).

26. Khemlina, G., Ikeda, S. \& Kurzrock, R. The biology of Hepatocellular carcinoma: implications for genomic and immune therapies. Mol Cancer 16, 149 (2017).

27. Fan, Z. et al. Hsp90\{beta\} andp130(cas): novel regulatory factors of MMP-13 expression in human osteoarthritic chondrocytes. Ann Rheum Dis 68, 976-982 (2009).

28. Torrungruang, $K$. et al. DNA binding and gene activation properties of the Nmp4 nuclear matrix transcription factors. J Biol Chem 277, 16153-16159 (2002).

29. Nakamoto, T. et al. Mice deficient in CIZ/NMP4 develop an attenuated form of K/BxN-serum induced arthritis. J Cell Biochem 117, 970-977 (2016). 